\title{
Efectividad del Programa PANTBC en el estado nutricional de pacientes con tratamiento antituberculoso
}

Effectiveness of the PANTBC program in the nutritional state of patients with tuberculosis treatment

\author{
Quesso-Flores, Alessandra Bethzabe ${ }^{1}$ \\ Vega-Gonzales, Emilio Oswaldo ${ }^{2}$ \\ Mosquera-Figueroa, Zoila ${ }^{2}$
}

\begin{abstract}
Resumen
Objetivo: determinar la efectividad del programa PANTBC en el estado nutricional de pacientes con tratamiento antituberculoso en la Microred Aeropuerto en el periodo 2018-2019. Materiales y métodos: estudio descriptivo longitudinal, en el que se evaluaron las medidas antropométricas y bioquímicas a todos los asistentes del Programa PANTBC. Se emplearon como técnicas la recolección de datos y la encuesta durante los seis meses de tratamiento de la enfermedad. Resultados: en las 30 personas que asistieron al programa se observó mejora en el IMC de 18,12 a $23,13 \mathrm{~kg} / \mathrm{m} \mathrm{2}$; y en los parámetros bioquímicos, especialmente la hemoglobina, que aumentó de $11,5 \mathrm{a} 13,8 \mathrm{~g} / \mathrm{dL}$ en mujeres y de 12,84 a 13,27 g/dL en varones. Se registraron dos meses en que no se entregó la canasta en toda la Microred. Conclusión: los parámetros antropométricos y bioquímicos mejoraron como resultado del tratamiento y aporte de la canasta PANTBC, aunque su distribución muestra irregularidad.
\end{abstract}

Palabras clave: Tuberculosis; Estado nutricional; Antropometría (Fuente: DeCS).

\begin{abstract}
Objective: to determine the effectiveness of the PANTBC program in the nutritional status of patients with tuberculosis treatment at Microred Airport in the 2018-2019 period. Materials and methods: longitudinal descriptive study, in which anthropometric and biochemical measurements were evaluated for all attendees of the PANTBC program. Data collection and survey techniques were used as techniques during the six months of treatment of the disease. Results: in the 30 patients who attended the program, there was improvement in the BMI from 18.12 to $23.13 \mathrm{~kg} / \mathrm{m} 2$; and in the biochemical parameters, especially hemoglobin, which increased from 11.5 to $13.8 \mathrm{~g} / \mathrm{dL}$ in women and from 12.84 to $13.27 \mathrm{~g} / \mathrm{dL}$ in men. Two months were recorded that the basket was not delivered throughout the Microred. Conclusion: anthropometric and biochemical parameters improved as a result of the treatment and contribution of the PANTBC basket, although its distribution shows irregularity.
\end{abstract}

Keys words: Tuberculosis; Nutritional Status; Anthropometry (Source: DeCS).

Para citar:

Quesso AB, Vega EO, Mosquera Z. Efectividad del programa PANTBC en el estado nutricional de pacientes con tratamiento antituberculoso. CASUS. 2019;4(3):187-193.

DOI: $10.35626 /$ casus.3.2019.214

\footnotetext{
${ }^{1}$ Universidad César Vallejo

${ }^{2}$ Escuela de Nutrición. Universidad César Vallejo

Correo electrónico: emilioswald@gmail.com
}

Fecha de recepción: 26-08-19

Fecha de envío a pares: 28-08-19

Fecha de aprobación por pares: 29-10-19

Fecha de aceptación: 15-11-19 


\section{INTRODUCCIÓN}

La tuberculosis (TB) es una enfermedad muy peligrosa debido a la facilidad con la que puede transmitirse, y afecta a gran parte de la población mundial, especialmente en aquellos países donde existe mayor índice de pobreza y pobreza extrema. Hacia el año 2016, se estimó que aproximadamente 11 millones de personas sufrieron de tuberculosis, de las cuales, 1.7 millones fallecieron como consecuencia de dicha enfermedad, presentándose la mayoría de estos casos en países en vías de desarrollo (1). Las personas que son diagnosticadas con TB suelen padecer alteraciones a nivel nutricional durante el tratamiento clínico, además de sufrir cuadros de depresión. El tratamiento para la tuberculosis también genera un gasto que afecta a la economía de los países que presentan una mayor tasa de prevalencia (2).

En algunos países latinoamericanos, como Chile, Costa Rica, Cuba y Uruguay, se ha logrado reducir la tasa de prevalencia de la TB a 20 casos por cada 100000 habitantes (3), lo cual se ha conseguido gracias a sus políticas implementadas, que consideran a esta enfermedad como un problema prioritario de salud pública (4). De estos países, Chile es el que presenta la cifra más baja de infectados, alcanzando en el año 2016 una tasa de 14 personas por 100000 habitantes, valor que no ha sufrido mayor variación en los últimos años (5).

En el Perú, las tasas de incidencia de TB y de morbilidad total por 100000 habitantes se han visto reducidas en un 2 a $3 \%$ anuales en el intervalo 2011-2015, pasando de un 97.4 a un 87.6 en el caso de la incidencia de TB, y de 109.7 a 99.5 en el caso de la morbilidad. En el caso de la tasa de mortalidad, esta ha tendido a aumentar durante el mismo periodo, pasando de una tasa de 2.9 a 3.7 por 100000 habitantes (6). Sin embargo, debe considerarse la existencia de un subregistro de casos, los cuales no son notificados de manera oportuna e incrementan el riesgo de que aparezcan complicaciones (7).

En torno a la alimentación, los hábitos alimentarios deficientes que presentan este grupo de pacientes suelen generar malnutrición, la cual afecta los indicadores del estado nutricional y la evolución del tratamiento antituberculoso. Esto se evidencia de manera más notoria en las zonas de la ciudad que presentan mayor hacinamiento y menor nivel socioeconómico. Por ello, el uso de suplementos nutricionales y actividades de educación nutricional, de manera simultánea al tratamiento antibiótico, contribuyen de manera significativa con el mejoramiento del paciente (8).

En el Perú el tratamiento con medicinas se complementa con estrategias de subvención de productos alimenticios. Esto se lleva a cabo a través del Programa de Alimentación y Nutrición al Paciente Ambulatorio con Tuberculosis y Familia (PANTBC), implementado por el Ministerio de Salud desde el año 2005, y que surge como parte del traspaso de los programas de alimentación complementaria que se encontraban a cargo del Programa Nacional de Asistencia Alimentaria (PRONAA), con el propósito de brindar soporte nutricional a las personas afectadas con TB para conseguir su recuperación integral en un corto plazo (9). Dicho programa consiste en la entrega mensual de una canasta con alimentos que permitiría cubrir el $50 \%$ de los requerimientos calóricos y proteicos de la persona afectada, así como de al menos cuatro familiares que viven con él, a fin de contribuir con la recuperación del paciente y la prevención del contagio a su entorno cercano. Los productos que se entregan en una canasta PANTBC son: 15 latas de leche evaporada entera, 15 latas de pescado enlatado e aceite, $15 \mathrm{~kg}$ de arroz blanco, $4 \mathrm{~kg}$ de maíz, $2 \mathrm{~kg}$ de quinua, $5 \mathrm{~kg}$ de lentejas, frejoles y arvejas, $4 \mathrm{~kg}$ de papa, 4 litros de aceite vegetal y $5 \mathrm{~kg}$ de azúcar rubia; con algunas variaciones de acuerdo a la región donde es distribuida (10). El Estado ha financiado este programa del Ministerio de Salud, mediante la Ley $\mathrm{N}^{\circ}$ 30518, Ley de Presupuesto del Sector Público para el Año Fiscal 2017, con un monto de hasta S/. 56000000 de soles (11).

El objetivo del presente estudio fue evaluar en qué medida varían los parámetros antropométricos y bioquímicos de los pacientes con tratamiento antituberculoso que participan en el programa 
PANTBC de la Microred Aeropuerto del Callao.

\section{MATERIALES Y MÉTODOS}

El estudio fue de tipo descriptivo longitudinal. La población estuvo formada por los 40 pacientes con tuberculosis atendidos en la Microred Aeropuerto, que comprende tres centros de salud: Aeropuerto, Playa Rímac y Polígono IV, pertenecientes a la Provincia Constitucional del Callao; y que participan en el Programa PANTBC. Del total de pacientes se excluyó al 25\% (10 casos), que abandonó el tratamiento y el Programa en el transcurso del estudio, quedando una muestra final de 30 pacientes. La principal razón por la que abandonaron el programa fue el incumplimiento con la entrega de las canastas ofrecidas.

Las técnicas de recolección de datos incluyeron la revisión documental y la observación. Se aplicó como primer instrumento una ficha de recolección de datos para evaluar en qué medida variaron los parámetros antropométricos y bioquímicos de los pacientes con tratamiento antituberculoso, durante el periodo de 6 meses, comprendido entre octubre del año 2018 hasta marzo del año 2019. El parámetro antropométrico empleado para el estudio fue el Índice de Masa Corporal (IMC) medido en $\mathrm{kg} / \mathrm{m}^{2}$, recolectado de manera mensual dentro de la última semana de cada mes; mientras que los parámetros bioquímicos incluyeron a la hemoglobina y las transaminasas (TGO y TGP), medidos en $\mathrm{mg} / \mathrm{del}$ y $\mathrm{UI} / \mathrm{L}$ respectivamente, y cuya medición fue realizada en tres momentos: al inicio del tratamiento, a los tres meses y a los seis meses, cuando el tratamiento termina. El segundo instrumento aplicado fue una lista de cotejo donde se verificó la entrega y distribución de las canastas PANTBC. Los instrumentos fueron validados mediante juicio de expertos, en el cual participaron tres magísteres en nutrición de la Universidad César Vallejo.

Para el análisis descriptivo de las variables del estudio se utilizó el programa estadístico SPSS versión 24, y para la elaboración de los gráficos correspondientes se empleó el programa Excel 2016.
Los pacientes firmaron un acta de consentimiento informado, en la cual se garantizó su participación anónima y el empleo de dicha información solo con fines del estudio.

\section{RESULTADOS}

En la tabla 1 se aprecia que, de los 30 pacientes que participaron en el estudio, la mayoría pertenecía al sexo masculino (60\%), tenía una edad entre 20 y 35 años (76.7\%), eran atendidos en el Centro de Salud Polígono (43.3\%), tenía tuberculosis pulmonar (96.7\%), con tratamiento TB-sensible (93.3\%) y un estado nutricional normal al inicio del tratamiento (63.3\%).

Tabla 1. Características generales de los pacientes con tuberculosis de la Microred Aeropuerto

\begin{tabular}{lcc}
\hline Característica & Frecuencia & Porcentaje \\
\hline Sexo & & \\
$\quad$ Masculino & 18 & $60.0 \%$ \\
$\quad$ Femenino & 12 & $40.0 \%$ \\
Edades & & \\
$\quad$ Menor de 20 años & 1 & $3.3 \%$ \\
$\quad$ Entre 20 y 35 años & 23 & $76.7 \%$ \\
$\quad$ Mayor de 35 años & 6 & $20.0 \%$ \\
Centro de Salud & & \\
$\quad$ C.S. Polígono & 13 & $43.3 \%$ \\
$\quad$ C.S. Aeropuerto & 10 & $33.3 \%$ \\
$\quad$ C.S. Playa Rímac & 7 & $23.3 \%$ \\
Tipo de TBC & & \\
$\quad$ Pulmonar & 29 & $96.7 \%$ \\
$\quad$ Extra pulmonar & 1 & $3.3 \%$ \\
Tratamiento & & \\
$\quad$ Sensible & 28 & $93.3 \%$ \\
$\quad$ Multirresistente & 2 & $6.7 \%$ \\
$\quad$ MDR) & & \\
Estado nutricional & & \\
$\quad$ Desnutrición & 1 & $3.3 \%$ \\
Delgadez & 4 & $13.3 \%$ \\
Normal & 19 & $63.3 \%$ \\
Sobrepeso & 6 & $20.0 \%$ \\
Total & 30 & $100.0 \%$ \\
\hline
\end{tabular}

Por su parte, en la figura 1 se observa que al inicio del Programa los pacientes tenían un bajo peso, con un IMC promedio de $18.12 \mathrm{~kg} / \mathrm{m}^{2}$, el cual mejoró considerablemente a un estado normal con un IMC de $23.13 \mathrm{~kg} / \mathrm{m}^{2}$.

En la tabla 2 se aprecia que los niveles de hemoglobina al inicio del tratamiento antituberculoso eran cercanos a los correspondientes a la anemia. Estos valores incrementaron luego de los seis meses de tratamiento, pasando de 11.55 a $13.80 \mathrm{~g} / \mathrm{dL}$ en 
mujeres y de 12.84 a $13.27 \mathrm{~g} / \mathrm{dL}$ en varones.

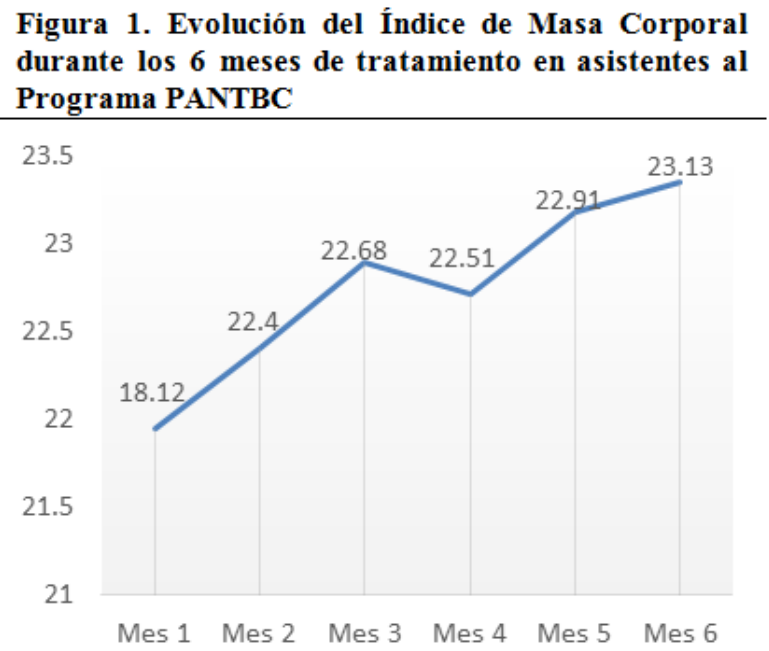

\section{DISCUSIÓN}

Los resultados obtenidos muestran que, al inicio del tratamiento, la mayoría de los pacientes con tuberculosis presentan un bajo IMC, con un promedio de bajo peso; resultado muy similar al reportado por otro estudio (12) con resultados que indicaron la presencia de desnutrición en la cuarta parte de los pacientes evaluados en cuanto a sus parámetros antropométricos. La mejora notoria del IMC a medida que avanza el tratamiento y se aplica el programa PANTBC, concuerda con otro hallazgo (13), que evidenció un mayor porcentaje de normopesos en cuanto a su IMC en los pacientes que participaban en dicho Programa, aunque este estudio fue de corte transversal y no permitió evidenciar la mejora del estado

Tabla 2. Variación de la hemoglobina según el sexo en asistentes al Programa PANTBC de la Microred Aeropuerto 2018 - 2019

\begin{tabular}{ccccccc}
\hline & \multicolumn{5}{c}{ Hemoglobina (mg/dL) } \\
Tiempo de & \multicolumn{3}{c}{ Femenino } & \multicolumn{3}{c}{ Masculino } \\
tratamiento & Min & Max & $\mathbf{m} \pm \mathbf{D E}$ & Min & Max & $\mathbf{m} \pm \mathbf{D E}$ \\
\hline 0 meses & 9.50 & 13.00 & $11.55 \pm 1.17$ & 10.00 & 15.84 & $12.84 \pm 1.29$ \\
3 meses & 10.00 & 13.60 & $11.99 \pm 1.15$ & 10.90 & 15.30 & $13.16 \pm 1.34$ \\
6 meses & 10.50 & 12.17 & $13.80 \pm 1.10$ & 11.90 & 15.20 & $13.27 \pm 0.99$ \\
\hline
\end{tabular}

Tabla 3. Variación de los niveles de transaminasas (TGO y TGP) en asistentes al Programa PANTBC de la Microred Aeropuerto 2018 - 2019

\begin{tabular}{|c|c|c|c|c|c|c|}
\hline \multirow{3}{*}{$\begin{array}{l}\text { Tiempo de } \\
\text { tratamiento }\end{array}$} & \multicolumn{6}{|c|}{ Niveles de transaminasas (UI/L) } \\
\hline & \multicolumn{3}{|c|}{ TGO* } & \multicolumn{3}{|c|}{ TGP** } \\
\hline & Min & Max & $\mathbf{m} \pm \mathbf{D E}$ & Min & Max & $\mathbf{m} \pm \mathbf{D E}$ \\
\hline 0 meses & 8.0 & 40.0 & $20.77 \pm 7.76$ & 7.5 & 40.0 & $19.95 \pm 9.72$ \\
\hline 3 meses & 11.0 & 102.0 & $37.05 \pm 27.44$ & 9.0 & 87.0 & $32.73 \pm 21.96$ \\
\hline 6 meses & 8.0 & 48.0 & $24.13 \pm 9.25$ & 9.0 & 28.0 & $17.32 \pm 5.90$ \\
\hline
\end{tabular}

Por otro lado, en la tabla 3 se observa que los niveles de transaminasas se encontraban dentro de los valores normales, y evidenciaron un aumento al terminar los tres meses de tratamiento, con valores máximos fuera de los límites normales, pero luego decrecieron notoriamente cuando se llegó a los seis meses. En el caso de las TGO, se encontró un aumento final en la media de 20.77 a $24.13 \mathrm{U} / \mathrm{L}$; mientras que, en las TGP, se evidenció una disminución final de 19.95 a 17.32 U/L.

En la figura 2 se observa que la distribución de la canasta PANTBC a los asistentes del Programa fue irregular, con ausencia de su entrega durante el tercer y cuarto mes del tratamiento, lo cual pudo afectar la efectividad de este.
Figura 2. Entrega de las canastas PANTBC a la Microred Aeropuerto durante el tiempo de evaluación del Programa

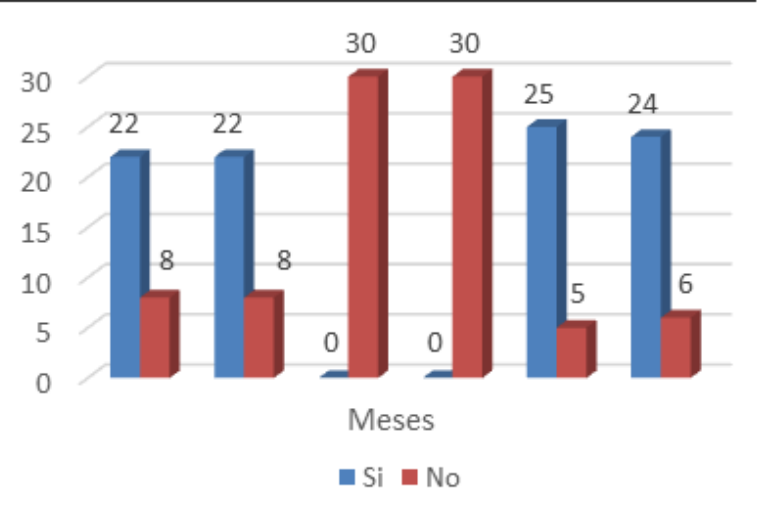


nutricional. Para la mejora de los indicadores antropométricos la distribución de alimentos a través de las canastas del Programa PANTBC juegan un rol importante, puesto que muchos de los pacientes tienen bajos recursos económicos y deficientes conocimientos que les impiden conseguir una dieta balanceada diaria.

En cuanto a los resultados de los parámetros bioquímicos, en la hemoglobina tomada a los asistentes se demuestra que, al iniciar el tratamiento, los pacientes presentaban un promedio propio de la anemia leve, la cual iba progresando de manera favorable conforme seguían el tratamiento hasta alcanzar un promedio normal. Sánchez (13), encontró un resultado similar en su investigación, con una mayoría de pacientes que presentaba anemia leve. Se destaca de dicho estudio la presencia de un nivel bajo de linfocitos, con cerca del $50 \%$ de pacientes con desnutrición según este parámetro, lo cual los predispone a sufrir infecciones, situación que se agrava aún más en pacientes que presentan enfermedades crónicas como la diabetes (14). Caso aparte lo constituyen los pacientes que son portadores del VIH, los cuales tienen un bajo nivel de conocimiento sobre la tuberculosis pulmonar, tal como fue indicado en un hospital público de Lima Norte (15).

Con respecto a las transaminasas TGO y TGP, estas al inicio del tratamiento, se encontraban dentro de los valores normales (16), pero en el tercer mes después de iniciado el Programa se incrementaron a casi el doble. Esto pudiera ser explicado debido a que el tratamiento farmacológico que se les daba a cada paciente incrementó en cuanto a los fármacos, ya que era crucial para la mejoría de ellos. En el último examen, tomado al finalizar el tratamiento, se observó que los valores regresaron a niveles cercanos a los iniciales demostrando que no hubo un daño hepático durante todo el proceso de curación de la tuberculosis (17).

La canasta PANTBC incluye una lista de 9 alimentos fundamentales para complementar el tratamiento farmacológico que conlleva la enfermedad. Su composición depende mucho de la región donde viva el paciente; por ejemplo, en la costa, la canasta incluye leche o lácteos, pescados en conservas, arroz, maíz, quinua, menestras, papa seca, aceite vegetal y azúcar. Cubre las necesidades de la persona afectada más cuatro personas del entorno familiar (10). La distribución de las canastas durante los seis meses que dura el tratamiento no muestra regularidad en la Microred Aeropuerto, evidenciándose la existencia de dos meses en los que no se cumplió con dicha entrega; pero aun así, el balance general fue positivo tanto en sus indicadores antropométricos como bioquímicos.

Al culminar el tratamiento se observó que los pacientes mejoraron notablemente beneficiando su estado nutricional, este hallazgo es corroborado por otro estudio (18) donde se observa el beneficio que aporta el Programa, sobre todo la canasta, PANTBC a todos sus asistentes. De igual manera al presente estudio las evaluaciones se realizaron frecuentemente con un mínimo de tres veces durante todo el tratamiento. Esto concuerda con lo expresado por otro reporte (18), a pesar de que solo un poco más del $24 \%$ usaron los servicios de nutrición, lo cual se explica porque el personal de salud no considera la consejería nutricional como parte del esquema de tratamiento y por la existencia de barreras de tipo administrativo para hacer la respectiva referencia.

La principal limitación del estudio es el bajo número de pacientes que formaron parte de este, debido entre otras causas, a la deserción del tratamiento indicado. Los resultados también pueden verse afectados por las deficiencias en la distribución de los productos de la Canasta PANTBC, lo cual no permitiría en los pacientes de bajos recursos económicos mantener una dieta balanceada.

\section{CONCLUSIONES}

La efectividad de la canasta PANTBC no fue la esperada ni la óptima, esto se debió a que durante el tercer y cuarto mes ningún asistente al Programa recibió la canasta afectando directamente su estado nutricional y también el desarrollo del estudio. En 
torno al IMC, se evidenció una progresiva mejoría en sus valores, alcanzado valores normales al terminar los seis meses de tratamiento. La variación de los parámetros bioquímicos fue adecuada, evidenciando una mejora en los niveles de hemoglobina, los cuales pasaron de un estado de anemia a valores normales; y en el caso de las transaminasas, un mantenimiento de los valores normales, lo cual indica el funcionamiento normal del hígado al terminar el tratamiento.
En base a estos resultados se recomienda una supervisión constante de la distribución de las canastas PANTBC, tanto en la entrega a los establecimientos de salud como a los pacientes beneficiarios. Asimismo, la evaluación del estado nutricional de estos pacientes debería ser mensual, a fin de corregir oportunamente cualquier alteración que pudiera producirse como resultado del tratamiento.

\section{REFERENCIAS BIBLIOGRÁFICAS}

1. Organización Mundial de la Salud. El informe sobre la TBC en el mundo 2014[internet]. Ginebra, Suiza: Centro de Prensa de la OMS, 2015. [citado el 25 de 6 . agosto del 2019] Disponible en: www.who.int/mediacentre/factshe ets/fs104/es/

2. Bermeo D., Gutierrez E. Evaluación del estado nutricional de los pacientes con tuberculosis pulmonar $\mathrm{Bk}+$, durante el periodo de tratamiento antifímico esquema I, zona siete, Ecuador [Tesis de Pregrado]. Ecuador: Universidad Nacional de Loja, 2016.

3. Organización Panamericana de la Salud. VI Reunión Regional de países de baja prevalencia de TB en las Américas [internet]. Santiago de Chile: Programa Regional de Tuberculosis, 2013. [citado el 30 de octubre del 2019] Disponible en: https://www.paho.org/hq/dmdocu ments/2014/2014-cha-bajaprevalencia-tb-Americas.pdf

4. Yáñez Á. Comentarios sobre los países de baja prevalencia de tuberculosis en América Latina. Revista Chilena de Enfermedades Respiratorias, 2013; 29(2): 112117.

5. Ministerio de Salud. Tuberculosis. Informe de situación Chile 2016[internet]. Chile: Minsal, 2016. [citado el 25 de agosto del 2019] Disponible en: https://diprece.minsal.cl/wrdprss_ minsal/wpcontent/uploads/2017/07/2017.07. 04_Infrome-Tuberculosis.pdf

Ríos J. Situación de Tuberculosis en el Perú y la respuesta del Estado (Plan de Intervención, Plan de Acción). Presentación en Power Point. [internet].Lima: Dirección de Prevención y Control de TBDPCTB Minsa, 2018. [citado el 30 de octubre del 2019] Disponible en:

http://www.tuberculosis.minsa.gob .pe/portaldpctb/recursos/20180605 122521.pdf

7. Alarcón V., Alarcón E., Figueroa C. y Mendoza A. Tuberculosis en el Perú: Situación Epidemiológica, Avances y Desafíos para su Control. Revista Peruana de Medicina Experimental y Salud Pública, 2017; 34(2): 300-301.

8. Islam Q., Ahmed S., Islam M., Kamruzzaman M., Rifat M. Beyond drugs: tuberculosis patients in Bangladesh need nutritional support during convalescence. Public Health Action, 2013; 3 (2):136-40.

http://bvs.minsa.gob.pe/local/MIN SA/1751.pdf

10. Ministerio de Salud. Documento técnico: formulación de la ración alimentaria del programa de complementación alimentaria para la persona afectada por tuberculosis [internet]. Lima, Perú: CENAN, 2015 [Citado el 25 de agosto del 2019] Disponible en: https://repositorio.ins.gob.pe/bitstr eam/handle/INS/311/CENAN0084.pdf;jsessionid=F280A9B2B3 3628BDAB90D9D417BC0861?se quence $=1$

11. Ministerio de Desarrollo e Inclusión Social. Directiva $\mathrm{N}^{\circ}$ 001-2017-MIDIS. Funcionamiento de la Modalidad de Complementación Alimentaria para la Persona Afectada por Tuberculosis-PANTBC del Programa de Complementación Alimentaria. Perú: Ministerio de Salud, 2017. [En línea]. Disponible en: https://busquedas.elperuano.pe/do wnload/url/aprueban-directiva-n001-2017-midis-funcionamientode-la-resolucion-ministerial-no025-2017-midis-1487381-1

Ministerio de Salud. Plan 12. Villanueva A, Guanche H, Álvarez Estratégico Multisectorial de la Respuesta Nacional a la Tuberculosis en el Perú 2010 2019 [internet].Lima, Perú: Ministerio de Salud. 2010. [citado el 25 de agosto del 2019] Disponible

en:
Y. Estado nutricional y rol de enfermería en pacientes con Tuberculosis pulmonar admitidos en el hospital cubano en Qatar. Tecno salud, 2016; 100(47):e. 
13. Sánchez G. Evaluación del estado 15. Núñez J., Mejía J. Factores nutricional de los pacientes con tuberculosis que asisten al centro de salud Perú Corea Bellavista, Callao, 2017 [Tesis de Pregrado]. Perú: Universidad César Vallejo, 2017.

14. Pérez LM, Fuentes J, Zenteno R. Tuberculosis pulmonar y diabetes mellitus tipo 2: el binomio perfecto. Univer Salud, 2012; 8(16):48-59. asociados a los conocimientos sobre tuberculosis pulmonar en pacientes con VIH. CASUS. Revista de investigación y casos en salud, 2019; 4(2):92-101. Carlos. Hígado y terapia antituberculosa. Revista chilena de enfermedades respiratorias,2011; 27(1):53-57.
16. Aguayo Miguel, Rodríguez Juan 18. Núñez G., Salinas A., Villarreal

17. Salazar E., Figueroa C., Machaca T. Variación del estado nutricional de los beneficiarios del programa PANTBC en Arequipa Metropolitana, 1996 - 2002. Revista Peruana de Medicina Experimental y Salud Pública, 2005; 22(2): 134-138. E., Garza E. y Gónzales F. Riesgo nutricional en pacientes con tuberculosis pulmonar: ¿cuestión del paciente o de los servicios de salud?. Salud Pública de México, 2000; 42(3):126-132. 